\title{
Transitional Structural Chemogenomics
}

\author{
N.J. Chan **, C.E. Gagna *,**, T. Yam** and W.C. Lambert* \\ * Department of Pathology, New Jersey Medical School, Newark, NJ \\ ** Department of Life Sciences, New York Institute of Technology, Old Westbury, NY
}

Advances in DNA, RNA and protein synthesis and sequencing have accelerated gene and protein discovery. Genomics, proteomics and their many subdisciplines are playing a greater role in molecular biology, cell biology and drug discovery and development. Merging disciplines have fostered advances in chemistry, molecular biology, genomics and proteomics. Nucleic acids are not static one-dimensional molecules. They are dynamic macromolecules that adopt many alternative conformations, undergo helical transitions and have multistranded DNA and RNA conformations [1]. Chemogenomics which targets genes to inhibit gene expression, has many limitations. It only targets the primary sequence (base pair) data. Transitional structural chemogenomics (TSCG) (Fig. 1) represents the next step in the evolution of chemogenomics [2-5]. Ultrasensitive small- (and large-) molecule drugs are used to target helical (conformational) transitions in the structure of DNA and RNA (e.g., B-DNA $\rightarrow$ Z-DNA, A-RNA $\rightarrow$ Z-RNA) [2-5]. Our novel method (TSCG) regulates, (i.e., turns "off," "on" or variably controls) (dimmer switch), the expression of normal or mutated genes [2-5]. As a result alternative nucleic acids (e.g., cruciform DNA) and other multistranded nucleic acids (e.g., triplex-DNA) can also be chemically targeted [1-4]. TSCG inhibits gene expression by using drugs targeted at regulatory regions of genes (e.g., enhancer) that undergo structural (helical) changes (e.g., right-handed B-DNA to left-handed Z-DNA). This prevents access of the regulatory DNA or RNA proteins to the nucleic acids. Fig. 1 shows how TSCG can inhibit gene expression and allow for quantifying drug binding to DNA. Sometimes, the variable enhancement of a gene would be favorable [2-6]. TSCG also targets specific differences within DNA (Z- and Z'-DNA). Our group is using three-dimensional models created by computer software to analyze DNA, RNA, and nucleic acid -ligand interactions. We have also developed the next generation of DNA, RNA and plasmid microarrays by immobilizing intact, unaltered nondenatured nucleic acids. TSCG and our novel multistranded DNA, RNA and plasmid microarray (figure1) can accelerate genomics and proteomics [6]. TSCG will help researchers to better understand DNA, RNA and proteins, treat diseases and discover new drugs with fewer side effects.

\section{References}

1. Sinden, R.R. ('94). DNA Structure and Function. Academic Press, New York.

2. Gagna, C.E., et al. ('05). Structural transitional chemogenomics and structural transitional chemoproteomics. FASEB J. 19, 521.4.

3. Gagna, C.E., et al. ('04). The effects of DNA and protein structure on cancer gene expression. Protein Sci. 13,152.

4. Gagna, C. E. ('04). Effects of nucleic acid structure on normal gene expression. J. Histochem. Cytochem. 52, S50.

5. Gagna, C. E., et al. ('04) Characterizing changes in the structure of normal ....... Mol. Biol. Cell. 15, 445a (2466).

6. Gagna, C. E. inventor: Gagna, C. E. and NYIT, assignees. Methods for Immobilizing Multistranded Nucleic Acid Molecules by Modifying More Than One Strand Thereof, And Binding Each Strand To A Solid Support. U. S. Patent No. 6,936,461 B2. Aug. 30, 2005. 


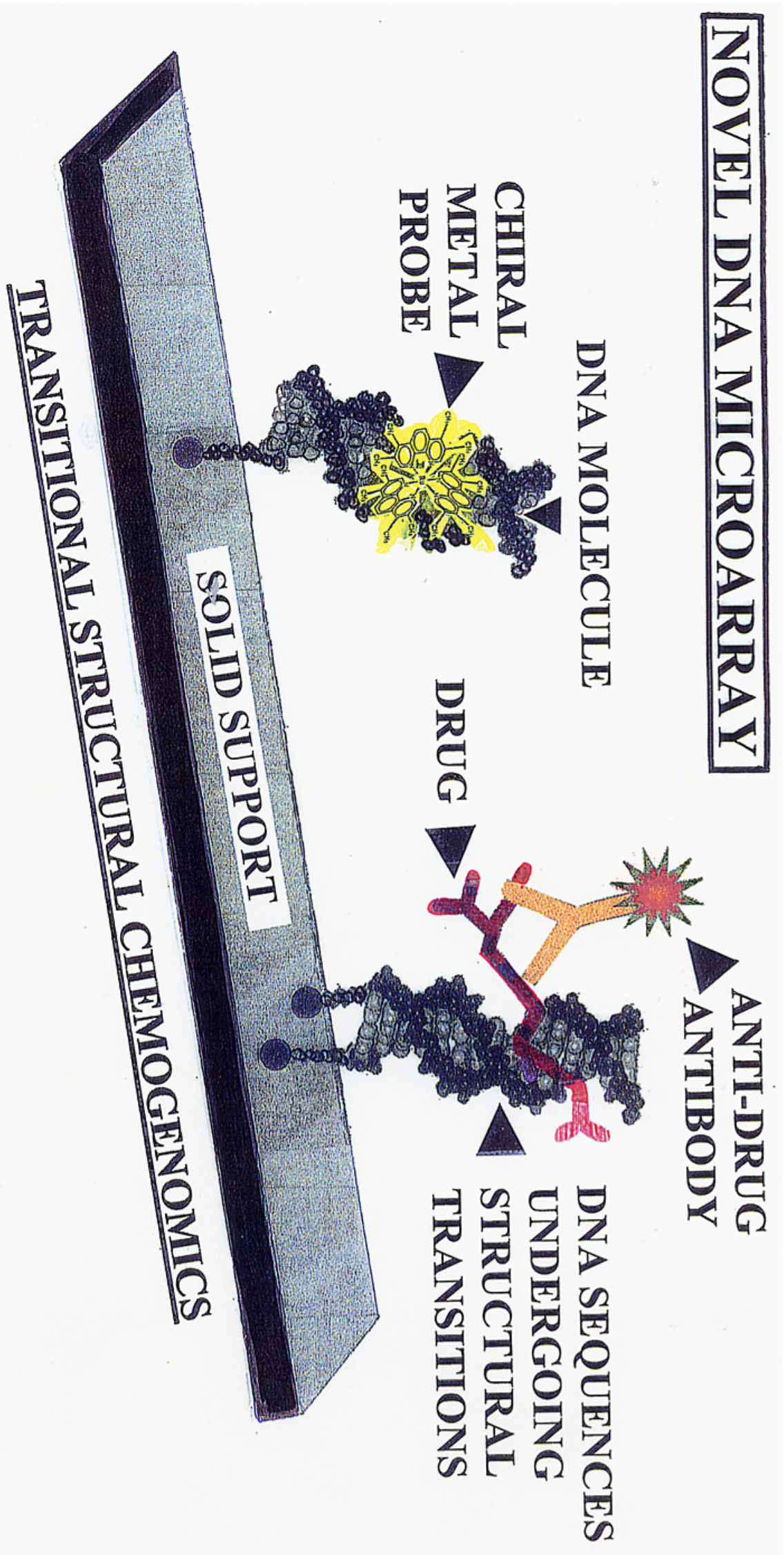


https://doi.org/10.1017/S143192760606257X Published online by Cambridge University Press 
https://doi.org/10.1017/S143192760606257X Published online by Cambridge University Press 
https://doi.org/10.1017/S143192760606257X Published online by Cambridge University Press 
https://doi.org/10.1017/S143192760606257X Published online by Cambridge University Press 